\title{
Extended reach spectrum-sliced passive optical access network
}

\author{
Bobrovs Vjaceslavs, Spolitis Sandis* and Ivanovs Girts \\ Institute of Telecommunications, Riga Technical University, Azenes Str. 12, Rīga, LV-1048, Latvia, Europe.
}

Accepted 8 April, 2013

\begin{abstract}
The spectrum sliced dense wavelength division multiplexed passive optical network (SS-DWDM PON) is a cost effective and power efficient solution for passive optical access networks to satisfy the growing worldwide demand for transmission capacity. In this work we successfully demonstrate the simulation model of effective spectrum-sliced 16-channel dense wavelength division multiplexed passive optical network (WDM-PON) system with data rate $2.5 \mathrm{Gbit} / \mathrm{s}$ per channel with significant optical link reach improvement by implementation of dispersion compensating fiber (DCF) and fiber Bragg grating (FBG) for chromatic dispersion compensation. We also show the realization of a broadband amplified spontaneous emission (ASE) light source with high output power $+23 \mathrm{dBm}$ and flat spectrum in wavelength range from 1545.32 to $1558.98 \mathrm{~nm}$ (C-band). Proposed system is built on ITU-T recommended DWDM frequency grid, in this way making it more adaptive and convergent to existing optical access systems.
\end{abstract}

Key words: Amplified spontaneous emission (ASE), arrayed-waveguide grating (AWG), optical fiber communication, spectrum slicing, wavelength division multiplexed passive optical network (WDM-PON).

\section{INTRODUCTION}

Spectrum sliced dense wavelength-division-multiplexed passive optical network (SS-DWDM PON) is an attractive and cost effective solution to satisfy the growing worldwide demand for transmission capacity in the next generation fiber optical access networks (El-Sahn et al., 2010; Spolitis and Ivanovs, 2011). Traditional wavelength division multiplexed (WDM) systems have multiple transmitter lasers operating at different wavelengths, which need to be wavelength selected for each individual channel operated at a specific wavelength (Vukovic et al., 2007). It increases complexity of network architecture, cost and wavelength (channel) management (Spolitis et al., 2011; Spolitis and Ivanovs, 2011). The strength of spectrum-sliced WDM-PON technology is use for one common broadband seed light source and its ability to place electronics and optical elements in one central office (CO), in that way simplifying the architecture of fiber optical network (Choi and Lee, 2011). Such optical systems benefit from the same advantages as WDM, while employing low cost incoherent light sources like amplified spontaneous emission (ASE) source or lightemitting diode (LED) (Kaneko et al., 2006; Bobrovs et al., 2011).

This spectral slicing method is promising and costefficient solution for the transmitter in an optical line terminal (OLT) of WDM-PON architecture. Spectrum sliced WDM PON system is more energy efficient than traditional WDM-PON systems because there is employed a common single broadband light source for transmission of large number of wavelength channels, 
not a one source per user as it is in the traditional WDMPON (El-Sahn et al., 2010). The optical bandwidth per channel of SS-WDM PON system is large compared to the bit rate. Therefore, dispersion considerably degrades the performance of this system more than it is observed in conventional laser-based systems (Choi and Lee, 2011). The influence of dispersion needs to be studied in order to understand the characteristics of a spectrum sliced WDM PON system employing standard single mode optical fiber.

The novelty of this paper is in fact, that we investigated the influence of chromatic dispersion compensation on overall improvement of multi-channel optical access system's performance as well as compared two different passive chromatic dispersion (CD) compensation techniques for network reach improvement.

Using the optical system's simulation experience from previous works (Spolitis et al., 2011; Bobrovs et al., 2011) we demonstrate a new model of 16-channel SS-DWDM PON system with CD compensation and broadband ASE seed light source with flat spectrum in wavelength range from 1545.32 to $1558.98 \mathrm{~nm}$ (C-band). In this system single broadband ASE source using flat-top type arrayedwaveguide gratings (AWG) are shared (sliced) among many user channels in such a way allocating a unique spectral slice to each user. AWG is mostly used as an optical filter because it can simultaneously play two roles as a filter and multiplexer (Bobrovs et al., 2011; Spolitis et al., 2012). In contrast to other studies made in this field before (El-Sahn et al., 2010; Choi and Lee, 2011) our main goal of this research is to evaluate and compare the reach improvement of SS-DWDM PON system by implementing dispersion compensating fiber (DCF) and fiber Bragg grating (FBG) for CD compensation in dispersion compensation module (DCM) as well as investigate the overall performance of this system by building it on the ITU-T DWDM frequency grid, defined in recommendation G.694.1.Based on this recommendation the channel spacing is chosen equal to $100 \mathrm{GHz}(0.8 \mathrm{~nm}$ in wavelength) (ITU-T Rec. G.694.1, 2012). In addition, our research differs from other researches made in this field with data transmission rate which is chosen 2.5 Gbit/s per channel, and is relatively high for this type of optical access networks. It is very important to build a new type optical system based on widely used frequency grid, recommended by international standards to make it more compatible with other already existing WDM-PON optical systems multiplexer (Spolitis et al., 2012). Our proposed spectrum-sliced dense WDM-PON system with CD compensation (where one seed broadband ASE source is spectrally sliced and used for multiple users) is potentially capable to replace existing classical WDMPON access system (where one laser source is used for each user) (Leeson et al., 2006; Leeson and Sun, 2008). The main benefit of this system includes the reduction of network architecture complexity as well as cost per one user.

\section{CHROMATIC DISPERSION COMPENSATION TECHNIQUES}

Chromatic dispersion significantly degrades the performance of spectrum sliced dense WDM PON system because of stochastic and noise-like nature of broadband ASE light source as well as large optical bandwidth of spectrum sliced channel (Choi and Lee, 2011). Dispersion causes optical signal pulses to broaden and lose their shape as they travel along the optical fiber. When pulses become wider, they have tendency to interfere with adjacent pulses. Eventually this limits the maximum achievable data transmission rate and transmission distance. The broadening of signal pulses causes intersymbol interference (ISI). Due to influence of ISI there can occur problems to restore transmitted information. In this case it is difficult to separate transmitted bit sequence at receiver side and it is resulting bit errors (high BER) or even failure of fiber optical transmission system (Spolitis et al., 2011; Bobrovs et al., 2011).

In order to reduce this negative impact DCM can be used for chromatic dispersion compensation and system's performance improvement in these fiber optical transmission systems (Kani et al., 2009; ITU-T Rec. G.694.1, 2012). Dispersion compensating fiber and fiber Bragg grating is used for CD compensation modules in our investigated SS-DWDM PON system.

Typically DCM is specified by what length, in $\mathrm{km}$, of standard single mode fiber (SMF) will be compensated or by the total compensation value of $C D$ over a specific wavelength range, specified in $\mathrm{ps} / \mathrm{nm}$. Dispersion compensation module can be placed before or after standard SMF span (CD pre-compensation or postcompensation). In our research we employ a precompensation configuration for effective chromatic dispersion compensation. In previous researches it is found as the most effective dispersion compensation configuration for WDM passive optical networks (Spolitis et al., 2011; Spolitis and Ivanovs, 2011).

DCM containing DCF fiber typically has high fiber attenuation and accordingly the insertion loss resulting from insertion of device in fiber optical transmission link will be high. DCM insertion loss can be compensated by optical amplifier, located in central office (CO). The effective core area $A_{\text {eff }}$ of a DCF fiber is much smaller than for standard ITU-T G.652 single mode fiber and thereby DCF experience much higher optical signal distortions caused by nonlinear optical effects (Chomycz, 2009). Typical DCF has a large negative dispersion and produces a negative slope. By lowering optical power the impact of nonlinear optical effects can be reduced. As a second solution for CD compensation are dispersion compensation modules with chirped FBG, (Figure 1). Because of good optical characteristics the chirped FBG is suitable for WDM systems.

Like DCF unit FBG is also a completely passive optical 


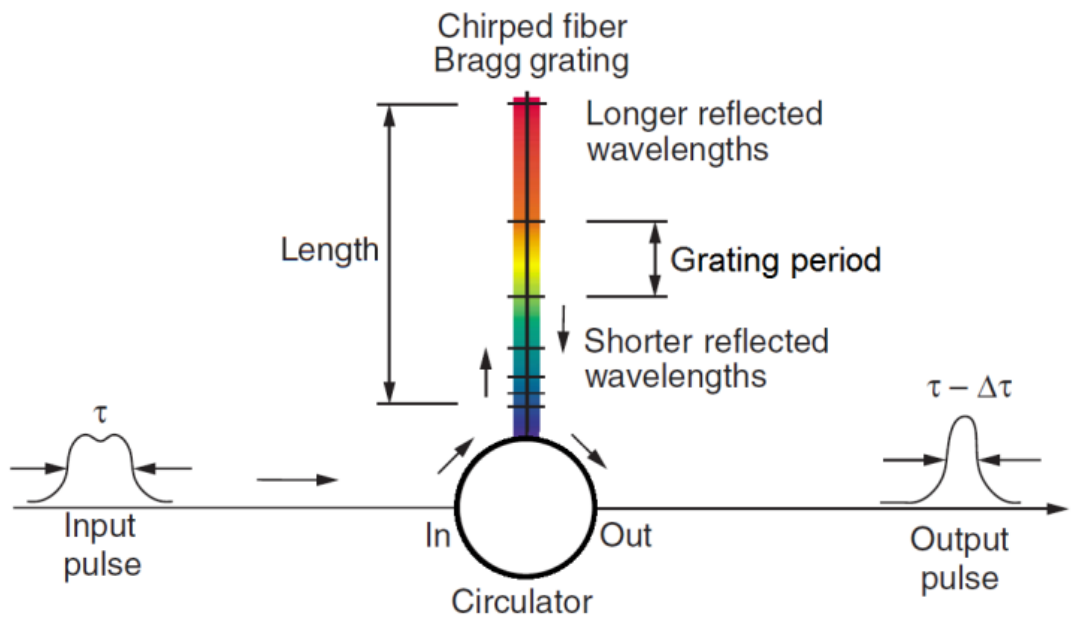

Figure 1. Chirped fiber Bragg grating.

unit. FBG has a grating period which is not constant but changes linearly over the length of the grating, starting with the shorter grating period located at the beginning of the grating. The grating period is a distance between two adjacent maximum values of the refractive index (Chomycz, 2009). The FBG reflects a narrow spectrum of wavelengths that are centered at $\lambda_{B}$ and passes all the other wavelengths. Reflected wavelength $\lambda_{B}$ can be obtained by the following equation:

$\lambda_{B}=2 \wedge n_{g}$

where $\lambda_{B}$ - reflected wavelength, $\mathrm{nm} ; \Lambda$ - grating period, $\mathrm{nm} ; n_{g}$ - fiber's effective group refractive index. When dispersion affected input pulse with width $T$ passes through the chirped FBG, pulse width is decreased by $\Delta T$ and its shape is restored on the output of transmission line.

FBG grating has shorter grating periods at beginning but over the length of the grating these periods linearly increases. It means that shorter optical signal wavelengths are reflected sooner and have a less propagation delay through the FBG unit but longer signal wavelengths travel further into the fiber grating before they are reflected back and accordingly have more propagation delay through the FBG. Typically the length of the FBG grating is from 10 to $100 \mathrm{~cm}$ (Agrawal, 2010).

A significant advantage of dispersion compensation modules with FBG over the DCF fiber is its relatively small insertion loss resulting from the insertion of a device in fiber optical transmission system. For example, insertion loss of commercially available DCF specified to compensate accumulated CD of up to $120 \mathrm{~km}$ standard single mode fiber span is about $10 \mathrm{~dB}$, whereas insertion loss of FBG based DCM capable to compensate the same CD amount is only up to $4 \mathrm{~dB}$ (Chomycz, 2009). In contrast to DCF DCM, the FBG based DCM can be used at higher optical powers without inducing nonlinear optical effects

\section{Spectrum-slicing technique of broadband ASE source using arrayed-waveguide grating}

One of basic techniques available in WDM PON systems in order to reduce the cost of components and simplify the passive network architecture is spectrum slicing technique where incoherent broadband light source (BLS) is used. Such a BLS is a good candidate for generating equally spaced multi-wavelength channels (Leeson and Sun, 2008; El-Sahn et al., 2010). The aim of spectrum slicing is to employ a single BLS for transmission on a large number of wavelength channels (Figure 2).

BLS is sliced using arrayed-waveguide grating (AWG). Afterwards optical slices are modulated, multiplexed by second AWG and transmitted over standard single mode optical fiber (SMF) line. Channels are demultiplexed by third AWG located in the end of fiber optical line and transmitted to optical receiver. This receiver can be PIN photodiode or avalanche photodiode (APD). Broadband light sources like LED, SLED or ASE can be used in spectrum sliced systems for data transmission. The transmission power available for each separate optical channel depends on the slice width. It should be considered that a larger slice will increase not only the total channel power but also increase the influence of dispersion and therefore the number of available WDM channels (El-Sahn et al., 2010).

In our research we choose ASE as BLS for spectral slicing because it has the highest optical output power compared to other mentioned broadband light sources. Optical signal from broadband ASE source is stochastic in nature and noise-like, therefore some assumptions and 


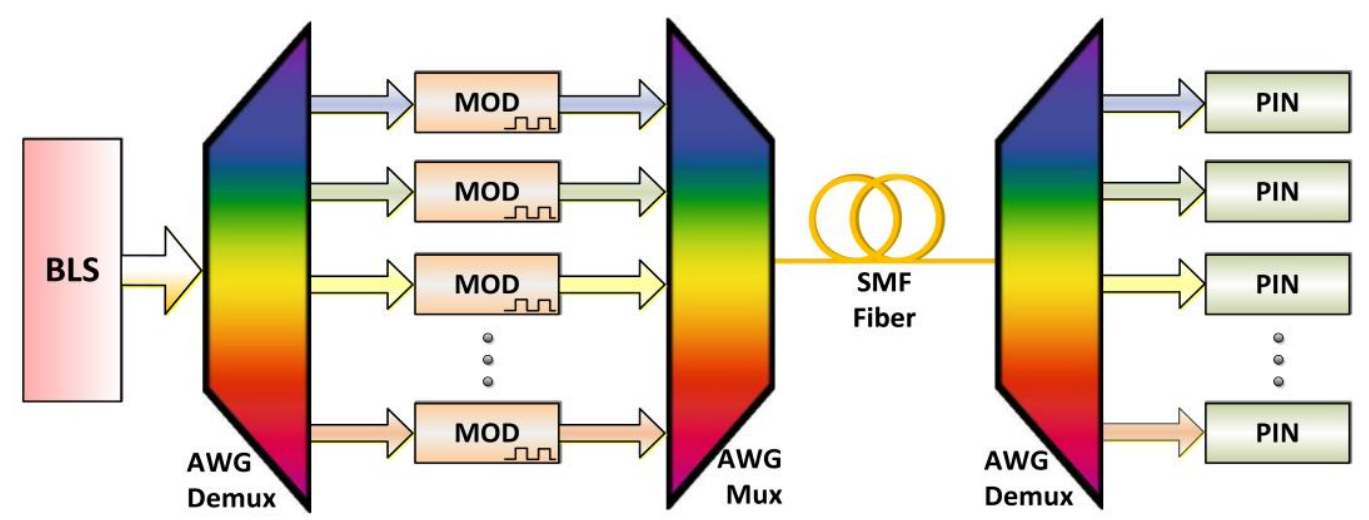

Figure 2. Operational principle of spectrum sliced system using broadband light source sliced by AWG demultiplexer.

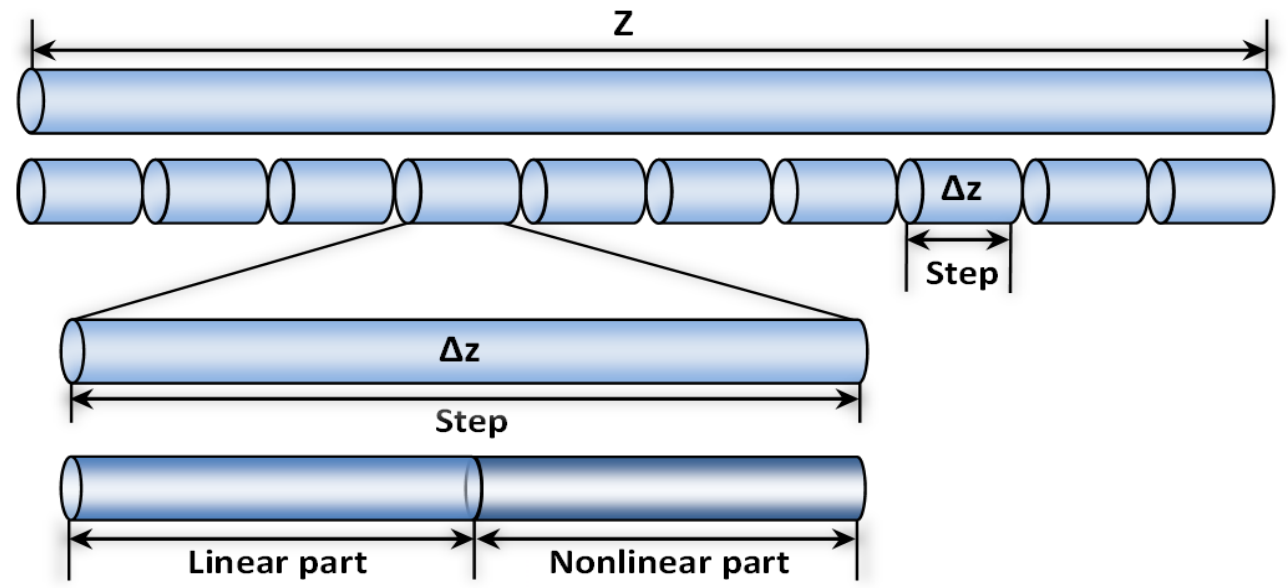

Figure 3. Illustration of time domain split-step (TDSS) algorithm.

formulations that is used in conventional laser based (e.g. distributed feedback (DFB) or Fabry Perot (FP) laser) passive optical systems may not be applicable for SSDWDM PON systems (Spolitis et al., 2012). In our research we investigate optical system where ASE spectral slicing is realized using AWGs.

\section{Numerical analysis and simulation model}

Our accepted research method is a mathematical simulation using newest OptSim 5.2 simulation software, where complex differential equation systems are solved using Split-Step algorithm. In order to study the nonlinear effects in optical fiber the nonlinear Schrödinger equation (NLS) is used. Except certain cases this equation cannot be solved analytically. Therefore, OptSim software is used for simulation of fiber optical transmission systems where it solves complex differential equations using Time Domain Split-Step (TDSS) method. This Split-Step method is being used in most commercial optical system simulation tools. The principle of the method is illustrated in Figure 3 and by the fiber propagation equation, which can be written as following:

$\frac{\partial A(t, z)}{\partial z}=\{L+N\} A(t, z)$

where $A(t, z)$ - optical field; $L$ - linear operator responsible for dispersion and other linear effects; $N$ - non-linear operator responsible for the nonlinear effects. It is assumed that linear $L$ and nonlinear $N$ effects affect the optical signal independently using the Split-Step method, if the span (step) of simulated optical fiber $\Delta z$ if enough small.

In Figure 3 is shown that each step $\Delta z$ consists of two half steps. In the first half step only linear effects (linear part) are taken into account, but in the second half step only nonlinear effects (nonlinear part) are taken into account. All optical fiber length $z$ is divided into steps $\Delta z$, and alternately linear and nonlinear effects are considered. For the most accurate results, it is necessary to carefully choose a step $\Delta z$. If this step $\Delta z$ is chosen too small, it will increase the time necessary to perform calculations, but if the step is chosen too large it will decrease accuracy of the calculations in our case the simulation of more than 1024 bits is made to achieve result's estimation accuracy not less than $95 \%$ 


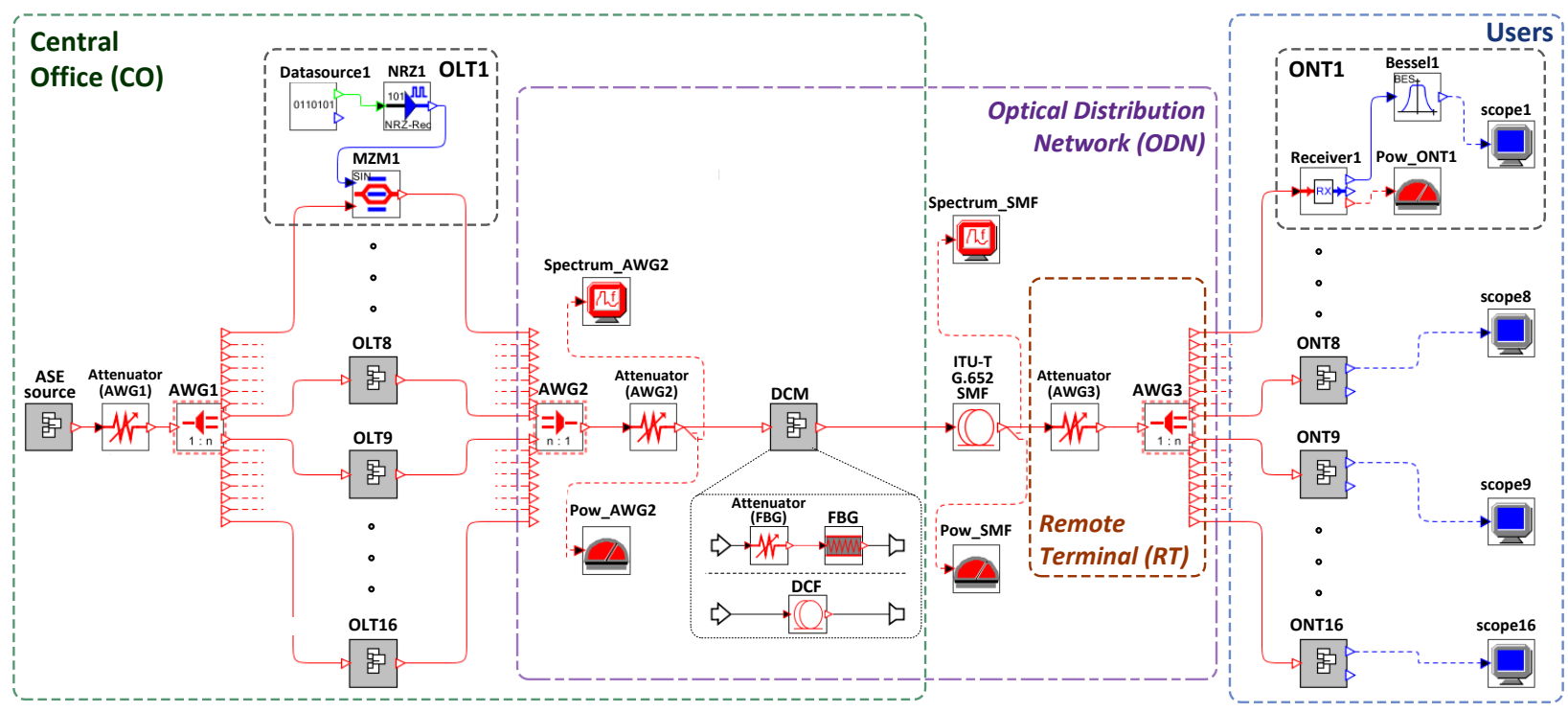

Figure 4. Simulation model of proposed high-speed 16-channel AWG filtered ASE seeded SS-DWDM PON system with DCM module.

(OptSim User Guide, 2012; Spolitis et al., 2011).

The performance of simulated scheme was evaluated by the obtained bit error ratio (BER) value of each WDM channel in the end of the fiber optical link. Basis on ITU recommendation G.984.2 it should be noticed that BER value for fiber optical transmission systems with data rate $2.5 \mathrm{Gbit} / \mathrm{s}$ per channel is specified less than $10^{-10}$ (ITU-T Rec. G.694.1, 2012).

In order to investigate the performance of incoherent broadband ASE light source as seed light for spectrum sliced WDM passive optical networks we created a simulation scheme of high-speed 16 channel SS-DWDM PON system (Figure 4). The design of high performance broadband flat spectrum ASE source on the output of cascaded EDFAs in details is described thus. This section describes the simulation model and parameters of proposed SSDWDM PON system with flat ASE seed source. As one can see in Figure 4, SS-WDM PON simulation scheme consists of 16 channels. The frequency grid is anchored to $193.1 \mathrm{THz}$ and channel spacing is chosen equal to $100 \mathrm{GHz}$ frequency interval (ITU-T Rec. G.984.2, 2003).

Broadband ASE light source is spectrally sliced using 16-channel flattop AWG filter (AWG1) with channel spacing equal to $100 \mathrm{GHz}$ $(0.8 \mathrm{~nm}$ in wavelength). ASE source average output power is 23 $\mathrm{dBm}$. Average output power of optical slices after AWG1 are about $5.8 \mathrm{dBm}$. Using this AWG unit we can obtain spectrally sliced optical channels (slices) with dense channel interval of $100 \mathrm{GHz}$. Insertion losses of AWG units are simulated using attenuation blocks (attenuators). Simulated a thermal high-performance AWG multiplexers and demultiplexers are absolutely passive optical components (no need for thermal regulation and monitoring electronics) with insertion loss up to $3 \mathrm{~dB}$ each (Spolitis and Ivanovs, 2011).

After spectrum slicing by AWG1 optical slices are transmitted to the optical line terminals (OLTs). OLTs are located at central office (CO). Each OLT consists of data source, non-return-to-zero (NRZ) driver, and external Mach-Zehnder modulator (MZM). Each MZM has $5 \mathrm{~dB}$ insertion losses, $20 \mathrm{~dB}$ extinction ratio, modulation voltage $V \pi$ of 5 Volts and maximum transmissivity offset voltage 2.5 Volts.

Generated bit sequence from data source is sent to NRZ driver where electrical NRZ pulses are formed. It has an electrical output signal which can assume one of the two electrical levels depending on the transmitted bit. When a "1" is fed into the driver, the output signal is at the low level during the entire bit time. When a " 0 " is fed into the driver, the output signal is at the high level during the entire bit time. Switching between the two levels may be instantaneous if the field Time Slope is set to zero, or not otherwise, with the desired time slope (OptSim User Guide, 2012). Used NRZ drivers have following parameters: low level -2.5 Volts, high level 2.5 Volts, time slope is 0 and crossing point is chosen $50 \%$. Afterwards formed electrical NRZ pulses are sent to MZM modulator. MZM modulates the optical slice from AWG1 and forms optical signal according to electrical drive signal. These formed optical pulses from all OLTs are coupled by AWG multiplexer (AWG2) and sent into standard optical single mode fiber (SMF) defined in ITU-T recommendation G.652.

Information from OLT is transmitted to an optical network terminal (ONT) or user over the fiber optical transmission link called optical distribution network (ODN). ODN consists of AWG multiplexer, two optical attenuators, dispersion compensation module (DCM), SMF with variable length and AWG demultiplexer. In our research we SMF with large effective core area Aeff $=80 \mu \mathrm{m}^{2}$, attenuation $\alpha=$ $0.2 \mathrm{~dB} / \mathrm{km}$, dispersion $\mathrm{D}=16 \mathrm{ps} /(\mathrm{nm} \cdot \mathrm{km})$ and dispersion slope Dsl $=0.07 \mathrm{ps} /\left(\mathrm{nm}^{2} \cdot \mathrm{km}\right)$ at the reference wavelength $\lambda=1550 \mathrm{~nm}$. As one can see in Figure 4 (middle), two simulated CD compensation methods implemented in DCM module are FBG and DCF. In our research we use DCF fiber with Aeff $=20 \mu \mathrm{m}^{2}$, attenuation $\alpha=0.55$ $\mathrm{dB} / \mathrm{km}$, dispersion $\mathrm{D}=-80 \mathrm{ps} /(\mathrm{nm} \cdot \mathrm{km})$ and dispersion slope $\mathrm{Dsl}=$ $0.19 \mathrm{ps} /\left(\mathrm{nm}^{2} \cdot \mathrm{km}\right)$ at the reference wavelength $\lambda=1550 \mathrm{~nm}$. Simulated FBG is tunable in terms of both reference frequency and total compensating dispersion value. Additional attenuator simulates $3 \mathrm{~dB}$ insertion loss of FBG unit. For the most accurate results we used real parameters of standard DCF fiber and tunable FBG in our simulation setup.

In the end of fiber optical link 16 optical channels are split using AWG demultiplexer (AWG3) located in remote terminal (RT). The receiver section includes ONT units. Each ONT consists of sensitivity receiver with PIN photodiode (sensitivity $\mathrm{S}=-25 \mathrm{dBm}$ at sensitivity reference error probability $\left.\mathrm{BER}=1 \cdot 10^{-10}\right)$, Bessel electrical lowpass filter (3-dB electrical bandwidth $B_{E}=1.6 \mathrm{GHz}$ ), 


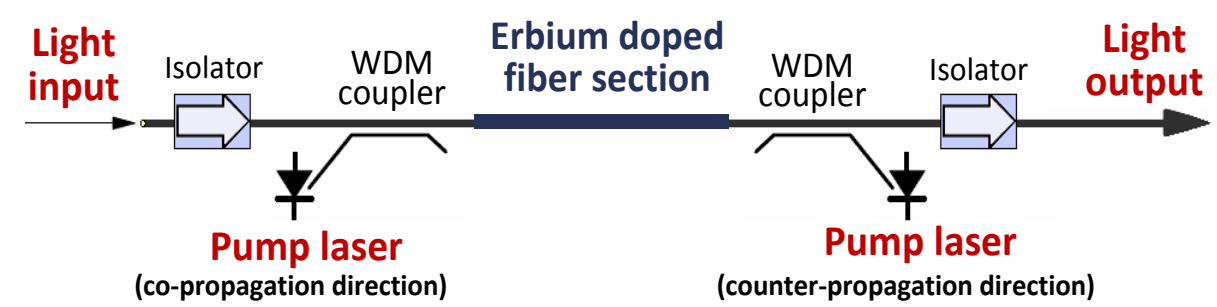

Figure 5. Scheme of erbium-doped fiber amplifier.

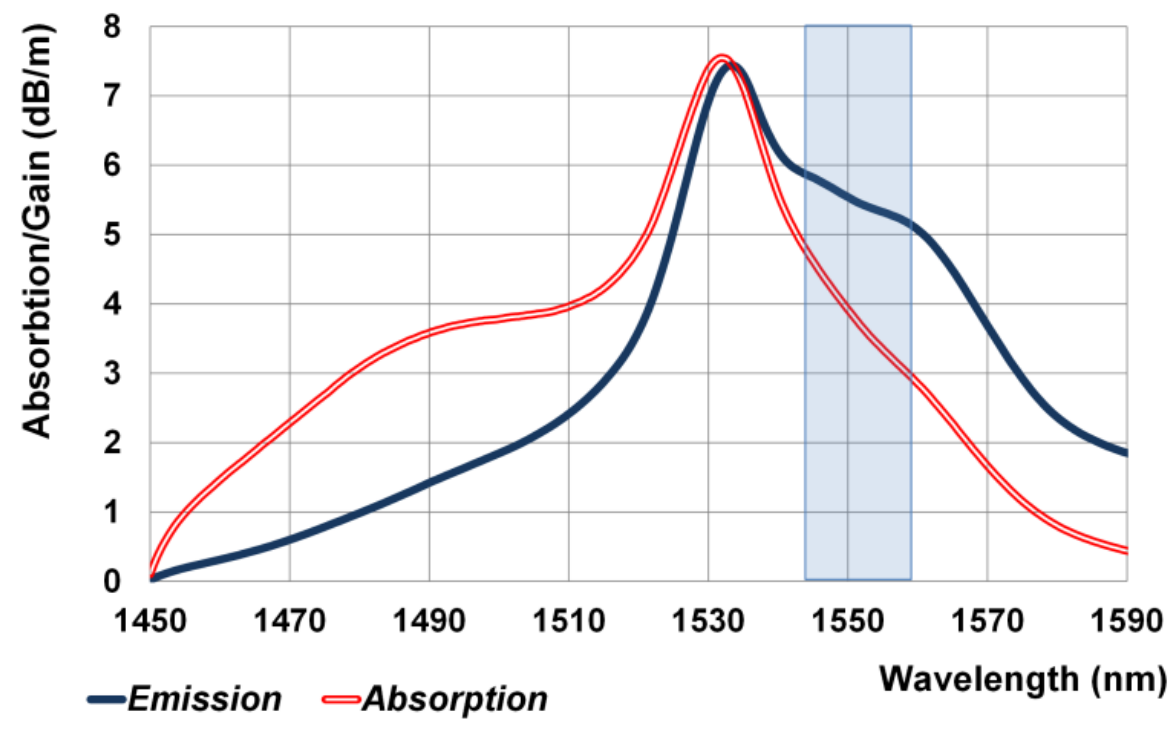

Figure 6. $\mathrm{Er}^{3+}$ emission and absorption spectrum in erbium doped fiber.

optical power meter and electrical probe to evaluate the quality of received optical data signal. Optical signal is converted to electrical signal using PIN photodiode and filtered by Bessel electrical filter to reduce noise (Keiser, 2003).

\section{Broadband ASE light source realization by erbium doped fiber amplifiers}

Erbium doped fiber amplifier (EDFA) consist of erbium-doped fiber having a silica glass host core doped with active $\mathrm{Er}^{3+}$ ions as the gain medium. Basic elements of an EDFA schematically are shown in Figure 5. Erbium-doped fiber is pumped by semiconductor lasers at 980 or $1480 \mathrm{~nm}$ in wavelength. By pumping on $980 \mathrm{~nm}$ we get low noise figure and low optical gain but pumping on $1480 \mathrm{~nm}$ we can get higher optical gain and also higher optical noise. Useful optical signal propagates along short span of a special erbium doped fiber and is being amplified at that time (Keiser, 2003; Agrawal, 2010).

Amplifier is pumped by a semiconductor laser (pump laser), which is coupled by a wavelength selective coupler, also known as WDM coupler. This WDM coupler combines the optical signal from pump laser with the transmitted optical signal which contains useful information need to been transmitted over the fiber optical link. The pump light propagates also in the same direction as the useful signal (co-propagation) or in the opposite direction (counter-propagation). Optical isolator is used to prevent laser oscillations and excess noise due to unwanted optical reflections (Keiser, 2003).

To make the principle work, erbium atoms needed to be set in excited state. This is done by $980 \mathrm{~nm}$ and/or $1480 \mathrm{~nm}$ lasers. The pump laser generates a high-powered optical signal beam at a wavelength on which erbium ions absorb and they get to their excited state. Pumping laser power is usually being controlled via feedback control circuit. It is known that EDFA emits high power amplified spontaneous emission (ASE) noise in C- and L- bands if there is no signal to be amplified (Agrawal, 2010). This effect is used to create broadband ASE light source in this research. ASE noise generation and gain occurs along all EDFA fiber length and it depends on erbium ion $\left(\mathrm{Er}^{3+}\right)$ emission and absorption spectrum shown in Figure 6. The marked area in Figure 6 shows the 1545.32 to $1558.98 \mathrm{~nm}$ wavelength range which is used for data transmission in our 16-channel spectrally sliced dense WDM-PON system. Broadband ASE light source realization can be done in different ways: by using one EDFA or connecting several amplifiers in cascade mode (one after another). The latter method allows achieving flatter ASE spectrum and higher output power because of better $\mathrm{Er}^{3+}$ ions usage along several amplifiers.

It should be taken into account that by default resulting ASE spectrum on the output of EDFA is not flat and manipulation with EDFA parameters must be done to make it plainer in range necessary for WDM system realization. Such parameters as EDFA 


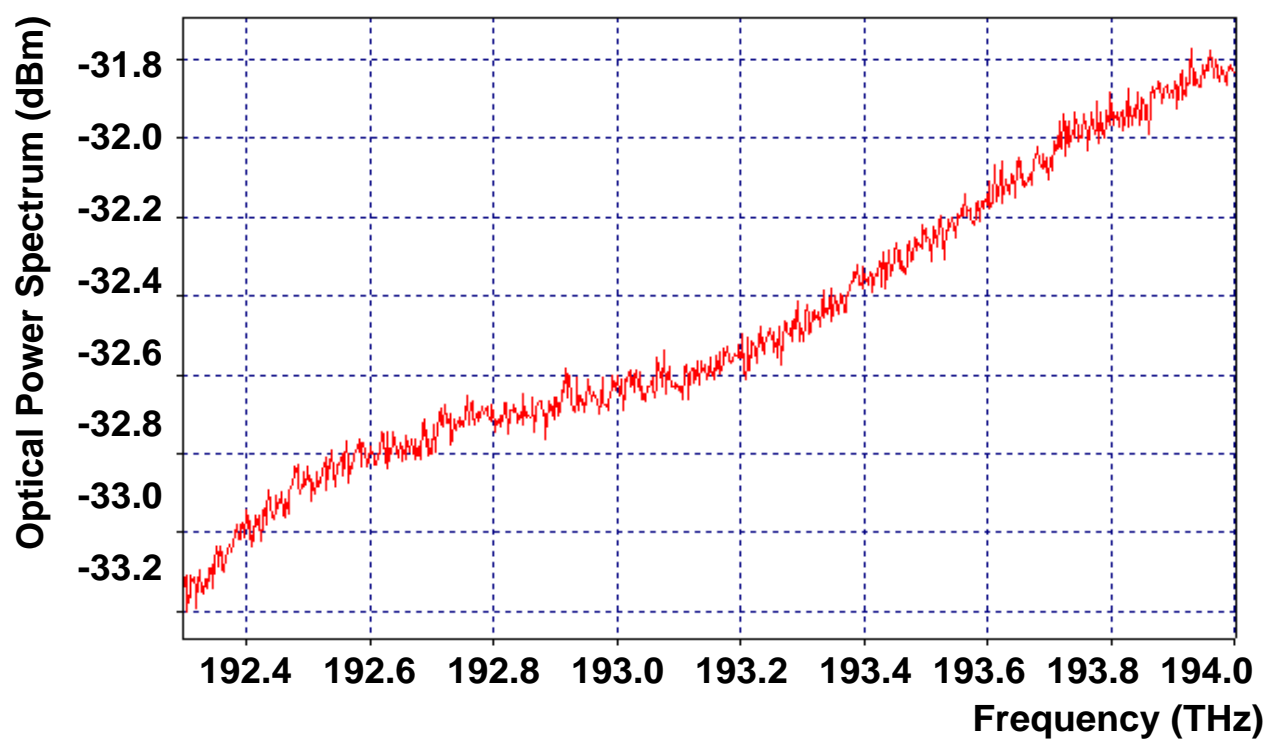

Figure 7. ASE spectrum after first EDFA in proposed SS-DWDM PON system's frequency range.

pumping laser power, its wavelength and length of erbium doped fiber affect $\mathrm{Er}^{3+}$ inversion - ratio between number of erbium ions in excited and ground state. This inversion has a direct impact on the shape resulting ASE spectrum. In our research the broadband ASE light source consists of two EDFAs combined in cascaded mode. First EDFA amplifier is realized using $9 \mathrm{~m}$ long erbium doped fiber and a $1480 \mathrm{~nm}$ co-directional pump laser with output power of 400 $\mathrm{mW}$. As it is seen in Figure 7, such power for the first EDFA is optimal to obtain flat output spectrum of the ASE on the output of cascaded EDFAs system.

First EDFA generates optical ASE signal with irregular spectrum in wide frequency range with total output power $-4.16 \mathrm{dBm}(0.38$ $\mathrm{mW}$ ). For our research we use the frequency range from $192.3 \mathrm{THz}$ to $193.4 \mathrm{THz}$ (wavelength range from 1545.32 to $1558.98 \mathrm{~nm}$ ), see marked area in Figure 6 . We investigate this particular frequency range because our proposed 16-channel spectrum-sliced dense WDM PON system operates in this range.

The spectrum of broadband ASE source from the first EDFA is being amplified and flattened using second EDFA. In our case the ASE spectrum on the output of EDFA can be flattened by achieving erbium ion inversion when lower frequencies in acquired spectrum are amplified more than higher ones. In this case it means that almost $100 \%$ of $\mathrm{Er}^{3+}$ ions inversion. For flattening of ASE output spectrum we used bi-directional laser pumping on both - 980 and $1480 \mathrm{~nm}$ wavelengths to uniformly distribute the energy along $12 \mathrm{~m}$ long erbium doped fiber section in the second EDFA amplifier. As can be seen in Figure 8 , by simultaneously increasing the pump power from 100 to $600 \mathrm{~mW}$ on all pump lasers (pumping on 1480 $\mathrm{nm}$ in first and pumping on 980 and $1480 \mathrm{~nm}$ in second EDFA) we obtained six optical output spectra of our ASE broadband light source.

The smoothest ASE broadband spectrum can be achieved if use $400 \mathrm{~mW}$ output power for all pump lasers, where first EDFA amplifier is pumped in co-propagating direction on $1480 \mathrm{~nm}$ wavelength, and second EDFA is pumped in both co-propagating direction on $1480 \mathrm{~nm}$ and counter-propagating direction on $980 \mathrm{~nm}$ (Figure 9).

In this manner we construct a broadband ASE source with almost flat spectrum and total output power on the output of cascaded EDFA system about $+23 \mathrm{dBm}(200 \mathrm{~mW})$. The spectrum of realized broadband ASE noise-like light source which can be spectrally sliced using AWG unit is shown in (Figure 10). The highlighted area in the figure shows the 1545.322 to $1558.983 \mathrm{~nm}$ wavelength range (centered on $1552.524 \mathrm{~nm}$ wavelength or $193.1 \mathrm{THz}$ in frequency) which is used for our 16-channel spectrally sliced DWDM-PON system. As one can see, fluctuations of optical power level are minimal and spectrum in this region is almost flat.

\section{RESULTS AND DISCUSSION}

There are compared two different CD compensation methods for improvement of maximal reach and performance of 16-channel AWG filtered SS-DWDM PON system with flattened ASE broadband light source. Flattop type AWG units were chosen for spectral slicing of ASE light source in our optical system because of good filtering performance, excellent WDM channel separation and bandwidth allow passing sufficient high optical power (El-Sahn et al., 2010; Spolitis et al., 2012). To reduce the negative impact of intensity noise as well as cross phase modulation the correct choice of filter's shape and 3-dB pass bandwidth is very important (Ozolins et al., 2010; Ivanovs et al., 2010). As a comparison in Figure 11 are shown two SS-DWDM PON channels (central frequencies 193.1 and $193.2 \mathrm{THz}$ ) which are filtered by Gaussian and flat-top type AWGs with $50 \mathrm{GHz} 3-\mathrm{dB}$ pass bandwidth.

It is shown that flat-top AWG has narrower spectral filter shape than Gaussian type AWG. Wider filter shape leads to larger slice width, higher resultant slice's power and optical signal dispersion (Leeson and Sun, 2008; Spolitis et al., 2012). In OptSim software we simulated flat-top type AWG filter shape using Raised Cosine optical filter's transfer function but using Super-Gaussian 


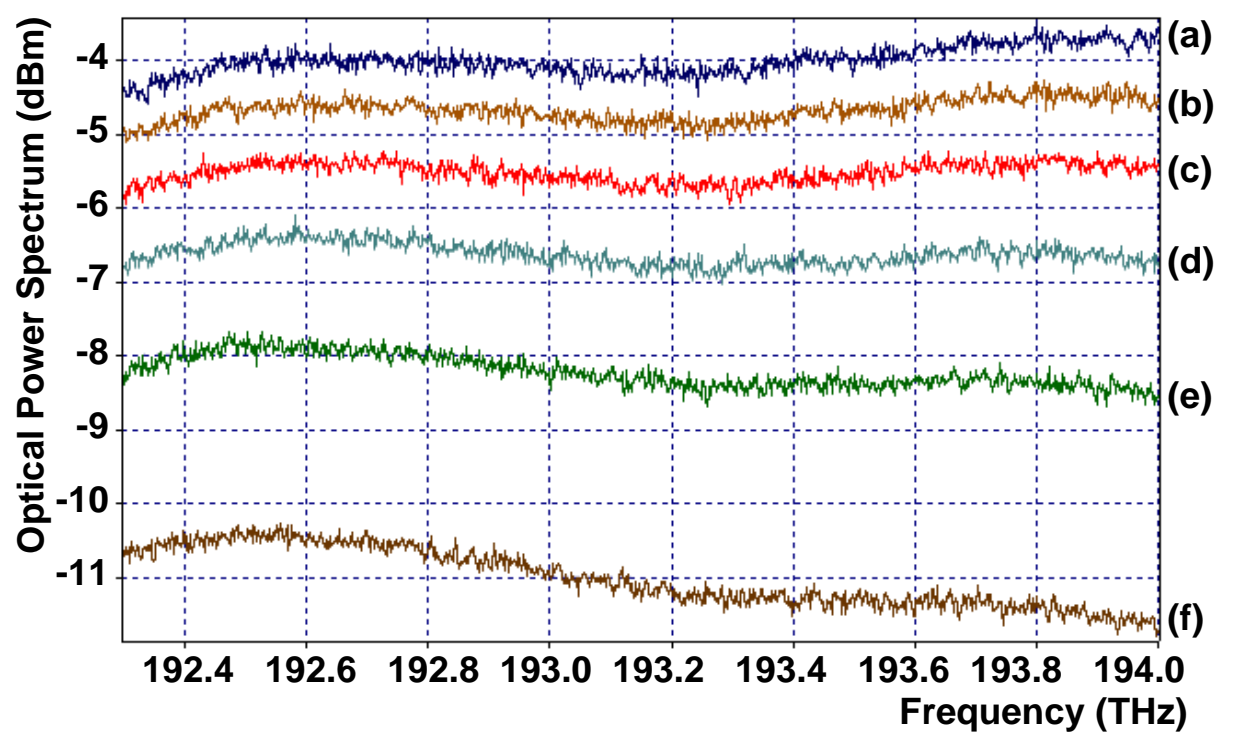

\section{Legend}
a) $-\mathrm{p}=600 \mathrm{~mW}$
b) $-p=500 \mathrm{~mW}$
c) $-p=400 \mathrm{~mW}$
d) $-p=300 \mathrm{~mW}$
e) $-p=200 \mathrm{~mW}$
f) $-p=100 \mathrm{~mW}$

Figure 8. ASE spectrum after second EDFA at different pump laser powers in proposed SSDWDM PON system's frequency range.

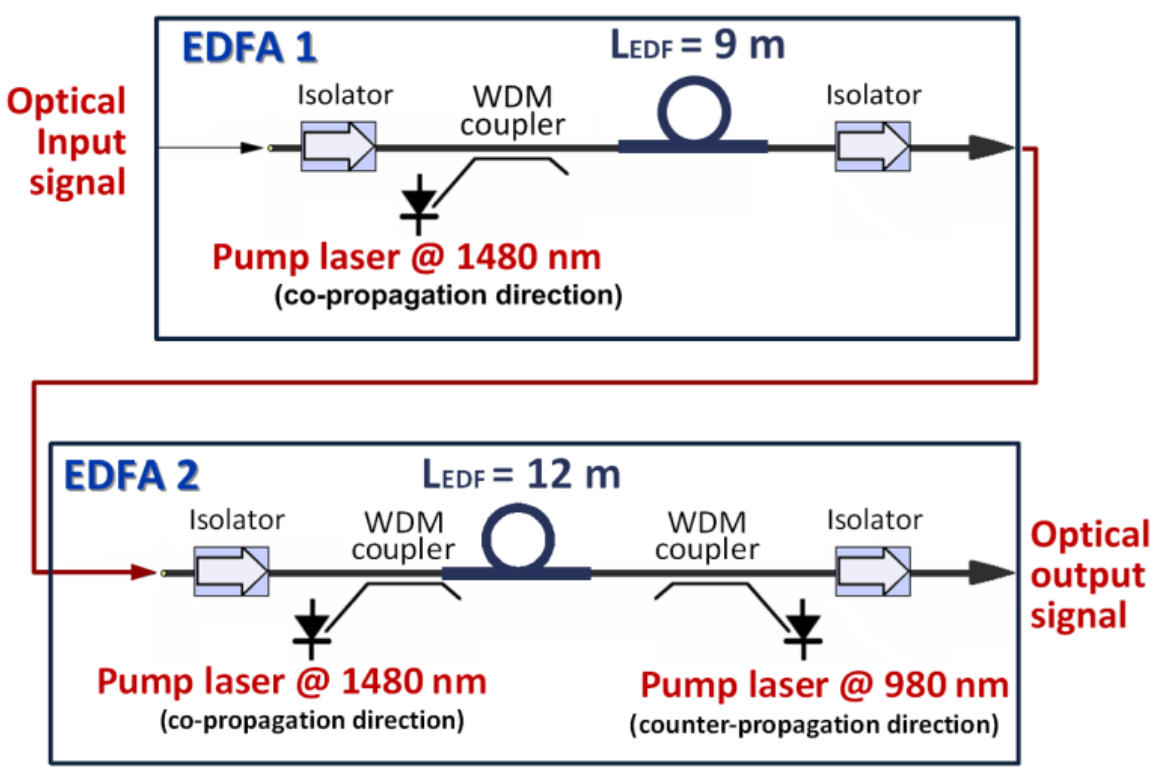

Figure 9. Detailed scheme of cascaded EDFA system with found parameters.

transfer function we approximate Gaussian AWG filter shape (Ozolins et al., 2010). As one can see in Figure 11, in the case of Gaussian filter the crosstalk between channels will be much higher than employing flat-top type filter. On the basis of this we can make a conclusion that AWG with flat-top filter spectral shape has higher optical signal to noise ratio (OSNR) than AWG with Gaussian type filter shape at identical bandwidth (3-dB bandwidth is 


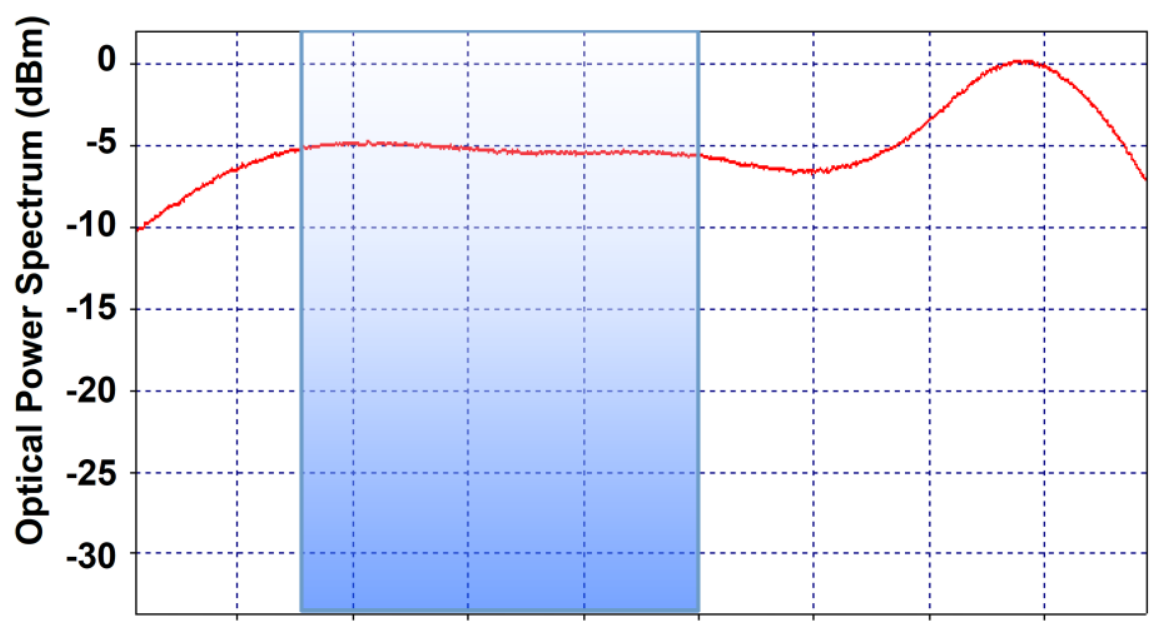

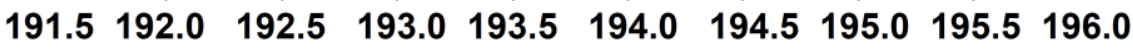

Frequency $(\mathrm{THz})$

Figure 10. Output spectrum of noise-like ASE broadband light source.

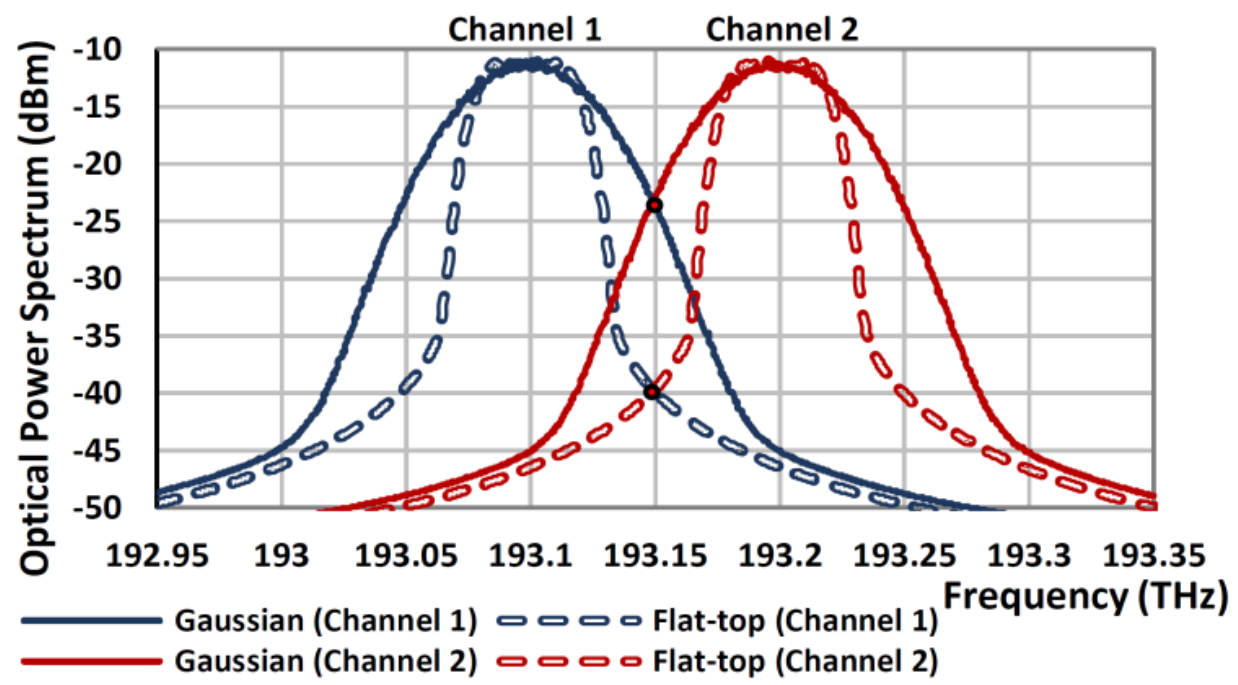

Figure 11. Comparison of Gaussian and flat-top type AWGs with $50 \mathrm{GHz} 3-\mathrm{dB}$ bandwidth in case of two adjacent WDM.

$50 \mathrm{GHz}$ ). Based on the above mentioned facts we chose flat-top AWGs for our investigated SS-DWDM PON simulation scheme.

Figure 12 shows the optical spectrum on the output of ASE source and spectra after each flat-top AWG unit. As one can see, flat-top AWG provides stable performance and good signal filtering on all stages. We found that optimal 3-dB bandwidth value of flat-top type AWG unit for maximal system performance must be about $90 \mathrm{GHz}$. By slicing the spectrum of proposed ASE source with AWG demultiplexer we obtain 16 separate WDM channels with channel output power variation less than
$0.42 \mathrm{~dB}$ that is very good result. As one can see in Figure 13 , the performance of investigated SS-DWDM PON system was completely sufficient to provide data transmission with BER $<1 \cdot 10^{-10}$ over the fiber optical span of $12 \mathrm{~km}$ in length without CD compensation. Theoretically, the value of accumulated CD in this span is about $190 \mathrm{ps} / \mathrm{nm}$. Figure 13 shows the worst channel after $12 \mathrm{~km}$ long fiber span we obtained BER $=8.47 \mathrm{e}-11$. The eye opening is good and system performance is sufficient to work under defined BER threshold.

The first realized CD compensation method includes the implementation of DCF in central office. It was found 


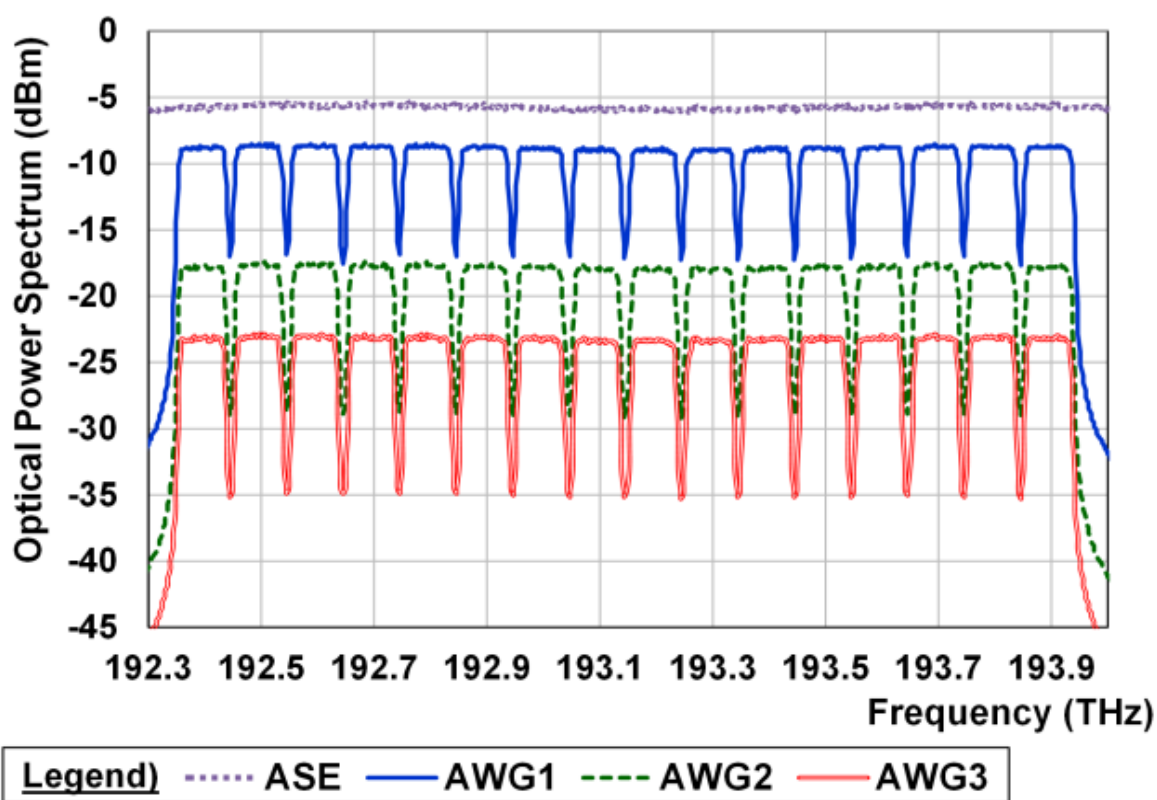

Figure 12. Optical output power spectrum of ASE source and 16-channel SS-DWDM PON system after each stage of flat-top type AWG unit.
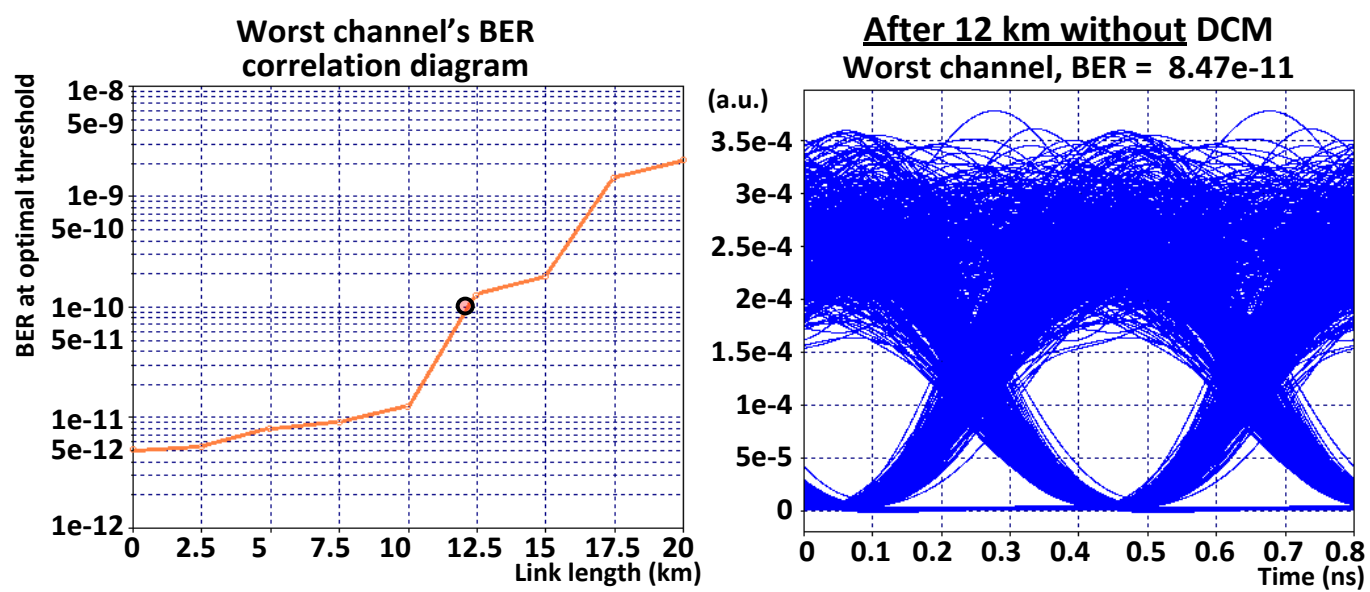

Figure 13. BER correlation diagram and output eye diagram of worst SS-DWDM PON channel after 12 km SMF link length without CD compensation module.

that optimal required DCF fiber length for maximum improvement of our SS-DWDM PON system's performance is $5.1 \mathrm{~km}$. This length of DCF fiber can compensate about $408 \mathrm{ps} / \mathrm{nm}$ of accumulated CD. Using DCF fiber with such a length we can achieve maximal 16channel SS-DWDM PON system's link length up to 24 $\mathrm{km}$ with $\mathrm{BER}=4.82 \mathrm{e}-11$ of received signal, (Figure 14a). Theoretical value of accumulated $C D$ in the span of 24 $\mathrm{km} \mathrm{SMF} \mathrm{is} \mathrm{about} 384 \mathrm{ps} / \mathrm{nm}$. By using FBG we achieve better results in the terms of network reach than using DCF for accumulated CD compensation. Implementation of $F B G$ for $C D$ compensation extends the total reach of
SS-DWDM PON system up to $26 \mathrm{~km}$ with BER=7.24e-11, (Figure 14b). Theoretical value of accumulated $C D$ in the span of $26 \mathrm{~km} \mathrm{SMF}$ is about $416 \mathrm{ps} / \mathrm{nm}$. We found that optimal CD compensation amount that must be compensated by FBG is $420 \mathrm{ps} / \mathrm{nm}$ and this amount is very close to full $C D$ compensation.

As one can see in Figure 14(a) and (b), that logical 1 level noise is larger than the logical 0 level noise which is due to an ASE beat noise generated by the spectrumsliced ASE BLS (Keiser, 2003). Using both CD compensation methods eye opening is wide enough for data transmission with $\mathrm{BER}<1 \cdot 10^{-10}$ over the fiber optical 


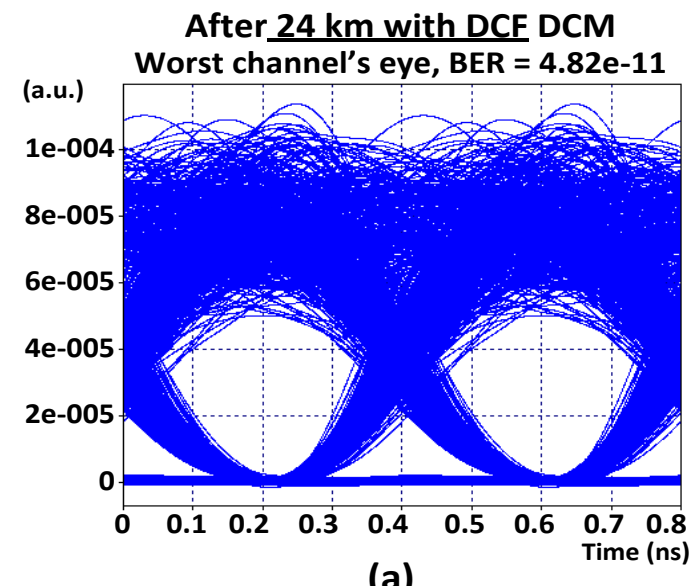

(a)

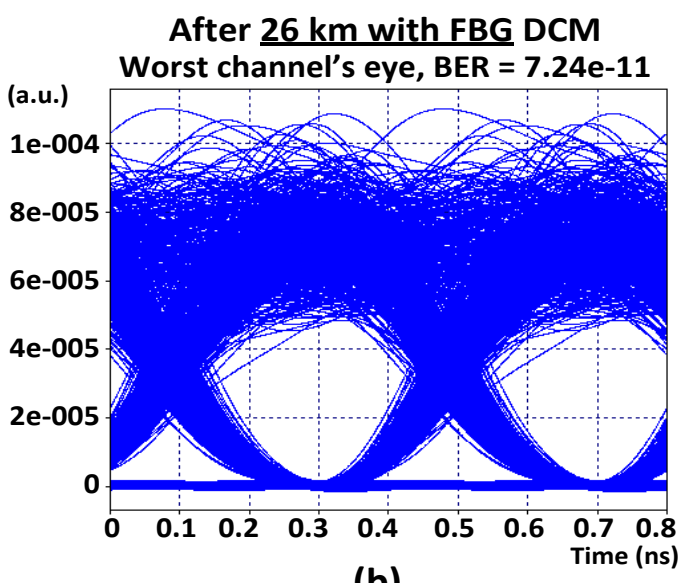

(b)

Figure 14. SS-DWDM PON system's eye diagrams and BER values of received signal from worst channels employing (a) DCF fiber and (b) FBG for accumulated CD compensation in DCM unit.

span. FBG provided greater performance improvement because it has relatively small insertion loss $(<4 \mathrm{~dB})$ and instead of DCF fiber it can be used at higher optical powers without inducing nonlinear optical effects (e.g. cross-phase-modulation-induced nonlinear phase noise (Grobe et al., 2013), which can reduce the system's performance.

\section{Conclusions}

In this work we have realized and investigated highspeed SS-DWDM PON system where DCF and FBG are used for accumulated chromatic dispersion (CD) compensation to improve the maximal link reach from OLT to ONT at the same time providing high system performance with BER $<1 \cdot 10^{-10}$. Design of broadband ASE source with $+23 \mathrm{dBm}$ output power and flat spectrum in system's operating wavelength range (Cband) using two EDFAs connected in cascade mode is shown and described.

We demonstrated that using DCF for CD compensation SS-DWDM PON reach can be improved by $100 \%$ or extra $12 \mathrm{~km}$ in length - from 12 to $24 \mathrm{~km}$. But using FBG unit network reach can be improved by $117 \%$ or extra 14 $\mathrm{km}$ - from 12 to $26 \mathrm{~km}$. On the basis of these results authors recommend to use FBG DCM units for CD compensation in future high-speed 16-channel dense SSWDM PON systems for maximal system's performance and network reach.

\section{ACKNOWLEDGMENT}

This work has been supported by the European Regional Development Fund in Latvia within the project Nr.2010/0270/2DP/2.1.1.1.0/10/APIA/VIAA/002.

\section{REFERENCES}

Agrawal GP (2010). Fiber-Optic Communication Systems, 4th edition. John Wiley and Sons, New Jersey, US. P. 626.

Bobrovs V, Udalcovs A, Spolitis S, Ozolins O, Ivanovs G (2011). Mixed chromatic dispersion compensation methods for combined HDWDM systems. Proc. IEEE Int. Conf. on Broadband and Wireless Comput. Commun. Appl. Barcelona. Spain. pp. 313-319.

Choi BH, Lee SS (2011). The effect of AWG-filtering on a bidirectional WDM-PON link with spectrum-sliced signals and wavelength-reused signals. Optics Commun. 284(24):5692-5696.

Chomycz B (2009). Planning Fiber Optic Networks. McGraw-Hill, New York. US. P. 401.

EI-Sahn ZA, Mathlouthi W, Fathallah H, LaRochelle S, Rusch LA (2010). Dense SS-WDM over legacy PONs: smooth upgrade of existing FTTH networks. J. Lightwave Technol. 28(10):1485-1495.

Grobe K, Searcy S, Tibuleac S (2013). The Costs of 10-Gb/s and 100$\mathrm{Gb} / \mathrm{s}$ Coexistence. The TERENA Networking Conference (TNC). Maastricht, Netherlands. pp. 1-3.

ITU-T Rec. G.984.2 (2003). Gigabit-capable passive optical networks (GPON): Physical media depend (PMD) layer specification. http://www.itu.int/rec/T-REC-G.984.2/en.

ITU-T Recommendation G.694.1 (2012). Spectral grids for WDM applications: DWDM frequency grid. http://www.itu.int/rec/T-RECG.694.1.

Ivanovs G, Bobrovs V, Ozolins O, Porins J (2010). Realization of HDWDM transmission system. Int. J. Phys. Sci. 5(5):452-458.

Kaneko S, Kani J, Iwatsuki K, Ohki A, Sugo M, Kamei S (2006). Scalability of spectrum-sliced DWDM transmission and its expansion using forward error correction. J. Lightwave Technol. 24(3):12951301.

Kani J, Bourgart F, Cui A, Rafel, A, Campbell M, Davey R, Rodrigues S (2009). Next-generation PON-part I: Technology roadmap and general requirements. IEEE Com. Mag. 47(11):43-49.

Keiser G (2003). Optical Communications Essentials. McGraw-Hill Networking Professional, New York. US. P. 372.

Lee K, Lim DS, Jhon MY, Kim HC, Ghelfi P, Nguyen T, Poti L, Lee SB (2012). Broadcasting in colorless WDM-PON using spectrum-sliced wavelength conversion. Optical Fiber Technol. 18(2):112-116.

Leeson MS, Luo B, Robinson AJ (2006). Spectral slicing for data communications. Proc. Symposium IEEE/LEOS Benelux Chapter, Eindhoven, pp. 165-168.

Leeson MS, Sun S (2008). Spectrum slicing for low cost wavelength division multiplexing. ICTON Mediterranean Winter Conference. Marrakech. Morocco. pp. 1-4.

OptSim User Guide (2012). RSoft Design Group Inc., New York. US. P. 459. 
Ozolins O, Bobrovs V, Ivanovs G (2010). Efficient wavelength filters for DWDM systems. Latvian J. Phys. Tech. Sci. 47(6):47-58.

Spolitis S, Ivanovs G (2011). Extending the reach of DWDM-PON access network using chromatic dispersion compensation. Proc. IEEE Swedish Communication Technologies Workshop, Stockholm, Sweden. pp. 29-33.

Spolitis S, Bobrovs V, Ivanovs G (2011). Realization of combined chromatic dispersion compensation methods in high speed WDM optical transmission systems. Elect. Elect. Eng. 10(116):33-38.

Spolitis S, Bobrovs V, Ivanovs G (2012). New Generation Energy Efficient WDM-PON System Using Spectrum Slicing Technology. 4th Int. Congress on Ultra Modern Telecommunications and Control Systems. St. Petersburg. Russia. pp. 558-562.
Vukovic A, Savoie M, Hua H, Maamoun K (2007). Performance Characterization of PON Technologies. Int. Conf. Appl. Photonics Technology, Photonics North. Ottawa, Ontario, Canada. P. 7. 